\title{
EXISTENCE AND NON-EXISTENCE FOR SCHRÖDINGER EQUATIONS INVOLVING CRITICAL SOBOLEV EXPONENTS
}

\author{
Henghui Zou
}

ABSTRACT. We study existence of positive solutions of the classical nonlinear Schrödinger equation

$$
\begin{aligned}
-\Delta u+V(x) u-f(x, u)-H(x) u^{2^{*}-1} & =0, \quad u>0 \quad \text { in } \mathbb{R}^{n} \\
u & \rightarrow 0 \quad \text { as }|x| \rightarrow \infty .
\end{aligned}
$$

In fact, we consider the following more general quasi-linear Schrödinger equation

$$
\begin{aligned}
& -\operatorname{div}\left(|\nabla u|^{m-2} \nabla u\right)+V(x) u^{m-1} \\
& -f(x, u)-H(x) u^{m^{*}-1}=0, \quad u>0 \quad \text { in } \mathbb{R}^{n} \\
& u \rightarrow 0 \quad \text { as }|x| \rightarrow \infty,
\end{aligned}
$$

where $m \in(1, n)$ is a positive number and

$$
m^{*}:=\frac{m n}{n-m}>0,
$$

is the corresponding critical Sobolev embedding number in $\mathbb{R}^{n}$. Under appropriate conditions on the functions $V(x), f(x, u)$ and $H(x)$, existence and non-existence results of positive solutions have been established.

\section{Introduction}

Let $n \geq 2$ be an integer and let

$$
V(x), H(x): \mathbb{R}^{n} \rightarrow \mathbb{R} \quad f(x, u): \mathbb{R}^{n} \times \mathbb{R} \rightarrow \mathbb{R}
$$

be real functions satisfying appropriate conditions to be specified later. Consider the following quasi-linear elliptic differential equation

$$
\begin{aligned}
& -\Delta_{m} u+V(x) u^{m-1}-f(x, u)-H(x) u^{m^{*}-1}=0, \quad u>0 \quad \text { in } \mathbb{R}^{n} \\
& u \rightarrow 0 \quad \text { as }|x| \rightarrow \infty \text {, }
\end{aligned}
$$

Received August 13, 2008; Revised December 23, 2008.

2000 Mathematics Subject Classification. Primary 35J20; Secondary 35J10.

Key words and phrases. concentration-compactness, critical Sobolev exponent, existence, $m$-Laplacian, minimax methods, mountain-pass lemma, Schrödinger equations. 
where $m \in(1, n)$ is a positive number and

$$
m^{*}:=\frac{m n}{n-m}>0,
$$

is the critical exponent of the Sobolev embedding $W^{1, m}\left(\mathbb{R}^{n}\right) \hookrightarrow L^{m^{*}}\left(\mathbb{R}^{n}\right)$, and

$$
\Delta_{m} \cdot=\operatorname{div}\left(|\nabla \cdot|^{m-2} \nabla \cdot\right)
$$

is the $m$-Laplace operator. When $m=2,(1.1)$ reduces to the classical nonlinear Schrödinger equation

$$
\begin{aligned}
-\Delta u+V(x) u-f(x, u)-H(x) u^{2^{*}-1} & =0, \quad u>0, \quad \text { in } \mathbb{R}^{n} \\
u & \rightarrow 0 \quad \text { as }|x| \rightarrow \infty .
\end{aligned}
$$

We are concerned with the question of existence of a non-negative and nontrivial function $u \in W^{1, m}\left(\mathbb{R}^{n}\right)$, called a solution of (1.1), satisfying (1.1). Exploiting the variational structure of (1.1), we shall employ the minimax methods to find suitable critical points of a functional corresponding to (1.1), which are in turn non-negative and non-trivial solutions of (1.1).

We begin with notations and assumptions.

As is custom, for a real measurable function $h(x)$ on $\mathbb{R}^{n}$, put

$$
h_{+}=h_{+}(x)=\max \{h(x), 0\}, \quad h_{-}=h_{-}(x)=\max \{-h(x), 0\} .
$$

For $t \in \mathbb{R}$, set

$$
\Omega_{t}:=\left\{x \in \mathbb{R}^{n} \mid V(x) \leq t\right\} .
$$

Throughout the entire paper, we assume that the functions $V(x), f(x, u)$ and $H(x)$ satisfy the following conditions, unless otherwise specified.

(V) There exists $\tau_{0}>0$ such that the set $\Omega_{\tau_{0}}$ has the finite Lebesgue measure. Moreover, $V \in L_{l o c}^{\infty}\left(\mathbb{R}^{n}\right)$ and there holds

$$
V_{0}:=\left(\int_{\mathbb{R}^{n}} V_{-}^{n / m}\right)^{m / n}<S
$$

where $S=S_{m}$ is the best constant for the Sobolev embedding

$$
W^{1, m}\left(\mathbb{R}^{n}\right) \hookrightarrow L^{m^{*}}\left(\mathbb{R}^{n}\right) .
$$

(H) $H \in C\left(\mathbb{R}^{n}\right)$ is non-negative and bounded from above, i.e.,

$$
0 \leq H(x) \leq H_{0}=\sup _{x \in \mathbb{R}^{n}} H(x)<\infty .
$$

(F) There exist constants $\beta>0$ and $\alpha_{1}, \alpha_{2} \in\left(m, m^{*}\right)$ such that $\beta\left(|u|^{\alpha_{1}}+|u|^{\alpha_{2}}\right) \geq u f(x, u) \geq \alpha_{1} F(x, u) \geq 0, \quad(x, u) \in \mathbb{R}^{n} \times \mathbb{R}$, where $F$ is the primitive of $f$

$$
F(x, u)=\int_{0}^{u} f(x, s) d s .
$$


Denote $W^{1, m}\left(\mathbb{R}^{n}\right)$ the Sobolev space of weakly differentiable functions on $\mathbb{R}^{n}$ equipped with the standard norm

$$
\|u\|_{1}:=\left(\int_{\mathbb{R}^{n}}|\nabla u|^{m}+\int_{\mathbb{R}^{n}}|u|^{m}\right)^{1 / m} .
$$

Let $E$ be the subspace of $W^{1, m}\left(\mathbb{R}^{n}\right)$ consisting of all elements satisfying

$$
\|u\|:=\left(\int_{\mathbb{R}^{n}}|\nabla u|^{m}+\int_{\mathbb{R}^{n}} V_{+}|u|^{m}\right)^{1 / m}<\infty .
$$

Then $E$ is a Banach space equipped with the norm (see Lemma 2.3, or [6])

$$
\|u\| \geq C\|u\|_{1}, \quad u \in E
$$

Consider the functional

$$
J(u)=\frac{1}{m} \int_{\mathbb{R}^{n}}\left(|\nabla u|^{m}+V|u|^{m}\right)-\int_{\mathbb{R}^{n}} F(x, u)-\frac{1}{m^{*}} \int_{\mathbb{R}^{n}} H|u|^{m^{*}}, \quad u \in E .
$$

Clearly $J \in C^{1}(E, \mathbb{R})$ and its Lagrange-Euler equation is (1.1) (actually for nonnegative critical points, which are precisely what we are looking for though). Therefore we turn to use the minimax methods of critical point theory to seek existence of non-negative and non-trivial critical points of $J$ in $E$, which are non-negative and non-trivial solutions of (1.1).

Let $\Phi$ be a real $C^{1}$ functional on a real Banach space $X$ and let $\mathcal{A}$ be a suitable class of subsets of $X$. By properly choosing the class $\mathcal{A}$, one may employ the minimax methods to characterize the so-called minimax values

$$
c:=\inf _{A \in \mathcal{A}} \max _{u \in A} \Phi(u),
$$

as critical values of $\Phi$, see for example [10] for more details.

In this paper we apply the well known mountain-pass lemma to obtain the existence of critical values (and critical points, of course) of $J$ given by (1.3), where the class $\mathcal{A}$ consists of all continuous paths from the origin to some point $v \in X(v \neq 0):$

$$
\mathcal{A}=\Gamma_{v}:=\{\gamma \in C([0,1], X) \mid \gamma(0)=0, \gamma(1)=v\},
$$

and hence

$$
c=c_{v}:=\inf _{\gamma \in \Gamma_{v}} \max _{u \in \gamma} \Phi(u) .
$$

To this end, one needs to choose a proper $v$ with some care. On the other hand, in applying a variational argument like the mountain-pass lemma, the so-called Palais-Smale, (PS) for short, condition plays a crucial role. Such a compactness requirement ensures the convergence of a so-called (PS) sequence $\left\{x_{j}\right\} \subset X$ which satisfies

$$
\Phi\left(x_{j}\right) \rightarrow c \in \mathbb{R}, \quad \Phi^{\prime}\left(x_{j}\right) \rightarrow 0 \text { in } X^{*} .
$$

It is known that the functional $J$ does not satisfy the (PS) condition in general, whence the mountain-pass lemma need not apply in general. This 
is due to the lacking of compactness in both the underlying space $\mathbb{R}^{n}$ and functional $J$ (i.e., the presence of the term $|u|^{m^{*}}$ with a critical growth). In the pioneer work [4], however, it was observed that certain functionals $\Phi$ may fail to satisfy the (PS) condition in general, but satisfy a local version of the (PS) condition. For example, a (PS) sequence $\left\{x_{j}\right\}$ of $\Phi$, under proper restriction, may be convergent if the values of the set $\left\{\Phi\left(x_{j}\right)\right\}$ are below a certain value $c_{0}$. Consequently, a minimax value $c<c_{0}$ can then be characterized as a critical value of $\Phi$. This observation was successfully used to establish existence of positive solutions for semi-linear boundary value problems involving critical Sobolev exponents on bounded domains in [4]. There the presence of a linear term $\lambda u$ is crucial to their arguments.

In a recent article [6], the authors considered the perturbed Schrödinger equations (4.9) with $m=2$. For all $\varepsilon>0$ sufficiently small, it was proved directly that there exists $c_{0}>0$ such that the corresponding functional $J_{\varepsilon}$ satisfies a local (PS) condition and, moreover, $J_{\varepsilon}$ possesses minimax values below level $c_{0}$. Consequently, all points with such small positive minimax values are critical points of $J_{\varepsilon}$, which are desired solutions of (4.9). The work [6] extends the classical work [4] to the entire space $\mathbb{R}^{n}$, where the lacking of compactness is severer: in both the functional $J_{\varepsilon}$ and underlying space $\mathbb{R}^{n}$. Again, the presence of the lower order non-linear term $f(x, u)$ (relative to the critical growth term $|u|^{m^{*}}$ ) plays a key role to compensate the lacking of compactness.

Turning back to (1.1), our first goal is to relax the requirements of [6] on the potential $V(x)$. In the meantime, we extend the work [6] to the quasi-linear analogue with a non-linear principal part $\Delta_{m}$. We have the following main existence result.

Theorem 1.1. There exists a positive number $\kappa_{0}=\kappa_{0}\left(n, m, \alpha_{1}, \alpha_{2}, \beta, \tau_{0}, H_{0}\right)$ $>0$ such that (1.1) admits a positive solution, provided that there exists $\phi_{0} \in E$ such that

$$
\max _{t \geq 0} J\left(t \phi_{0}\right)<\kappa_{0}, \quad \liminf _{t \rightarrow \infty} J\left(t \phi_{0}\right)<0 .
$$

The proof of Theorem 1.1 is based on the ideas used in the works $[4,6]$. As mentioned earlier, a key ingredient is to show that (PS) sequences of $J$ converge (up-to a sub-sequence) if their minimax levels are below a certain level $\left(\kappa_{0}\right.$ given in (1.6) in our case). Here we also use the concentration-compactness principle due to $[7,8]$ (see also [17] and the references therein for further developments), which remains effective for the non-linear $m$-Laplacian operator $\Delta_{m}$. In our arguments, both the presence of the lower order term $f(x, u)$ and the behavior of the potential $V(x)$ are crucial. To illustrate this notion, we present three examples of existence, namely, Corollaries 4.1-4.3. In this regard, existence of a positive solution can hold for suitable potentials $V(x)$, even with $f(x, u) \equiv 0$, see Corollary 4.3. On the other hand, three non-existence results (Theorems 5.1-5.3) are established to further demonstrate the importance of 
the presence of the lower order term $f(x, u)$ and the behavior of the potential $V(x)$ to existence.

We would like to use the following canonical model of (1.1) to illustrate our results:

$$
-\Delta_{m} u+V(x) u^{m-1}-b u^{\alpha}-u^{m^{*}-1}=0, \quad u>0, \quad \text { in } \mathbb{R}^{n},
$$

where $b \geq 0$ and $\alpha \in\left(m-1, m^{*}-1\right)$ are constant. We consider several cases.

A) Take $b=1$ and let $R_{0}$ be the quantity given in Corollary 4.1. Set

$$
V(x)= \begin{cases}h(x), & |x| \leq 2 R_{0}, \\ 1, & |x|>2 R_{0},\end{cases}
$$

where $h(x)$ is any real bounded measurable function. Then, by Corollary 4.1 , there exists $\varepsilon>0$ such that (1.1) has a positive solution, as long as

$$
\sup _{x \in B_{2 R_{0}}(0)}|h(x)|<\varepsilon .
$$

B) For $\mu>0$ and $\lambda>m / 2$, let

$$
V(x)=-\mu\left(1+|x|^{2}\right)^{-\lambda} .
$$

Then, by Corollary 4.3, there exists $\varepsilon>0$ such that (1.1) has a positive solution, as long as

$$
\mu<\varepsilon, \quad b=0
$$

C) For $\mu>0$, let

$$
V(x)=-\mu\left(1+|x|^{2}\right)^{-m / 2} .
$$

Then (1.1) has no positive solutions for any $\mu>0$ and $b>0$. This is a direct consequence of Theorem 5.3.

D) For applications of Theorem 5.3 to positive potentials, we take $V(x)=$ $|x|^{-\lambda}$ with $\lambda \in[m, n)$. Note that Theorem 5.3 still applies, though the differentiability of $V$ needs to be modified slightly. Then, by Theorem 5.3, (1.1) has no positive solutions for any $b>0$.

E) Take $b=0$ and $V(x)=$ Const. $>0$. Then, by Theorem 5.2, (1.1) has no positive solutions.

Case E) is well-known. But, to the best knowledge of the author, Cases A)D) are new, even for the Laplacian operator $(m=2)$. It is worth adding that similar arguments can be applied to derive multiplicity results of [6] and one may treat hardy-type potentials $V(x)$ by using the Hardy-Littlewood-Sobolev inequalities.

The paper is organized as follows: we give some preliminary discussions in Section 2. A local version of the (PS) condition is established for the functional a corresponding to (1.1) in Section 3. In Section 4, we prove the main Theorem 1.1 and its Corollaries 4.1-4.3. Finally, the proofs of non-existence Theorems 5.1-5.3 are given in Section 5. 


\section{Preliminaries}

Let $E$ be the Banach space introduced in Section 1. For convenience, we write

$$
L(u)=\int_{\mathbb{R}^{n}}\left(|\nabla u|^{m}+V|u|^{m}\right), \quad N(u)=\frac{1}{m^{*}} \int_{\mathbb{R}^{n}} H|u|^{m^{*}}, \quad u \in E .
$$

Plainly $L(u)$ corresponds to the 'linear' part $-\Delta_{m} u+V u^{m-1}$ of (1.1) and

$$
J(u)=\frac{1}{m} L(u)-N(u)-\int_{\mathbb{R}^{n}} F(x, u) .
$$

Recall for $w \in E$,

$$
c=c_{w}:=\inf _{\gamma \in \Gamma_{w}} \max _{u \in \gamma} J(u),
$$

where

$$
\Gamma_{w}:=\{\gamma \in C([0,1], E) \mid \gamma(0)=0, \gamma(1)=w\},
$$

being the set of all continuous paths from the origin to $w$.

In order to obtain a positive solution, we need to modify the 'non-linear' part of $J$ as follows

$$
J_{1}(u)=\frac{1}{m} L(u)-N\left(u^{+}\right)-\int_{\mathbb{R}^{n}} F\left(x, u^{+}\right),
$$

where $u^{+}=\max \{u, 0\}$. It turns out that all the calculations below carried on $J$ equally apply to $J_{1}$ and so do the results. Therefore, for notational convenience, we shall remain working on the functional $J$, but later apply the results to $J_{1}$ at a proper time.

The first result in this section is the following mountain-pass lemma without the (PS) condition for $J$, due to Ambrosetti-Rabinowitz, see [1, 4].

Lemma 2.1 (Mountain-pass Lemma). Assume that there exist a neighborhood $U$ of the origin 0 in $E$, an element $v \in E \backslash U$ and a constant $\rho$ such that

$$
\left.J\right|_{\partial U} \geq \rho,
$$

$$
J(0)<\rho, \quad J(v)<\rho .
$$

Then there is a sequence $\left\{u_{j}\right\}$ in $E$ such that

$$
J\left(u_{j}\right) \rightarrow c=c_{v} \geq \rho, \quad J^{\prime}\left(u_{j}\right) \rightarrow 0 \text { in } E^{*} .
$$

Recall that a sequence $\left\{u_{j}\right\}$ in $E$ satisfying

$$
J\left(u_{j}\right) \rightarrow c, \quad J^{\prime}\left(u_{j}\right) \rightarrow 0 \text { in } E^{*}
$$

is called a (PS) sequence at level-c. The quantity $c$ is then called a critical-level of $J$.

It is well-known that the best constant

$$
S=S_{m}=n\left(\frac{n-m}{m-1}\right)^{m-1}\left(\frac{\Gamma(n / m) \Gamma(n+1-n / m) \boldsymbol{\omega}_{n-1}}{\Gamma(n+1)}\right)^{m / n}
$$


for the Sobolev embedding is achieved by the functions

$$
\phi(x)=\left(\kappa+|x|^{m /(m-1)}\right)^{(m-n) / m}, \quad \text { i.e., } \quad S\left(\int_{\mathbb{R}^{n}}|\phi|^{m^{*}}\right)^{m / m^{*}}=\int_{\mathbb{R}^{n}}|\nabla \phi|^{m},
$$

where $\boldsymbol{\omega}_{n-1}$ is the area of the sphere $S^{n-1}$ and $\kappa>0$, see for example [2, 13].

The next lemma is the concentration-compactness principle due to Lions $([7,8])$. Without further mentioning, in the rest of the paper, all convergencies may be up to a sub-sequence.

Lemma 2.2 (Concentration-compactness principle). Let $\left\{u_{j}\right\}$ be a bounded sequence in $E$ converging weakly and a.e. to some $u_{0} \in E$. Then there exist two bounded non-negative measures $\lambda$ and $\mu$ on $\mathbb{R}^{n}$ such that

$$
\left|\nabla u_{j}\right|^{m} \rightarrow \mu, \quad\left|u_{j}\right|^{m^{*}} \rightarrow \lambda
$$

in the sense of measure. In addition, there exist an index set $I$ and a set of points $\left\{z_{i}\right\} \subset \mathbb{R}^{n}(i \in I)$ such that

A) Either $I$ is an empty set (then so is $\left.\left\{z_{i}\right\}\right)$, or

B) There exists a positive integer $N$ such that

$$
\text { either } I=Z \quad \text { or } \quad I=\{1, \ldots, N\} \text {, }
$$

where $Z$ is the set of all natural numbers. Moreover, there exist two corresponding sets of positive numbers $\left\{\lambda_{i}\right\}$ and $\left\{\mu_{i}\right\}$ such that

$$
\lambda=\left|u_{0}\right|^{m^{*}}+\sum_{i \in I} \lambda_{i} \delta_{z_{i}}, \quad \mu \geq\left|\nabla u_{0}\right|^{m^{*}}+\sum_{i \in I} \mu_{i} \delta_{z_{i}}
$$

where $\delta_{z}$ is the Dirac measure at $z$, and there holds

$$
S_{m} \lambda_{i}^{m / m^{*}} \leq \mu_{i}, \quad i \in I .
$$

Remark. The quantity $\int_{\mathbb{R}^{n}}\left|u_{j}\right|^{m^{*}}$ is sometimes called the 'energy' level of $u_{j}$. It turns out that the limiting energy-level, $\lim _{j \rightarrow \infty} \int_{\mathbb{R}^{n}}\left|u_{j}\right|^{m^{*}}$, of the sequence $\left\{u_{j}\right\}$ in Lemma 2.2 may split into three possible parts as follows:

1) (Regular Part) This part of energy is represented by the quantity $\int_{\mathbb{R}^{n}}\left|u_{0}\right|^{m^{*}}$.

2) (Singular Part) This part of energy is concentrated on the countable set $\left\{z_{i}\right\}$ of finite singularities in terms of the Dirac measure.

3) (Escaping Part) Finally, part of energy may escape at infinity, which is represented by

$$
\lambda_{\infty}=\lim _{R \rightarrow \infty} \lim _{j \rightarrow \infty} \int_{\mathbb{R}^{n}}\left|u_{j}\right|^{m^{*}} \eta_{R}
$$

where $\eta_{R}$ is a suitable cut-off function at infinity, see Section 3 for details. 
All three possibilities 1-3) above could occur. Moreover, the sequence $\left\{u_{j}\right\}$ converges to $u_{0}$ in $L^{m^{*}}\left(\mathbb{R}^{n}\right)$ if and only if its limiting energy-level is given by the regular part. That is, no energy is concentrated on finite singularities, or escaping at infinity, see also [17] and the references therein.

To conclude this section, we show that every (PS) sequence of $J$ is bounded in $E$. In what follows, we use $C$ to denote positive generical constants depending only on structure constants, i.e., $n, m, \alpha, \ldots$, etc., which may vary from one to another. For $s>0$ and $x \in \mathbb{R}^{n}$, denote $B_{s}(x)$ the ball centered at $x$ with radius $s$.

Lemma 2.3. There exist $d_{0}, d_{1}>0$ depending only on the structural constants such that

$$
\|u\|^{m} \geq L(u) \geq d_{0}\|u\|^{m} \geq d_{1}\|u\|_{1}^{m}, \quad u \in E .
$$

Moreover, every (PS) sequence $\left\{u_{j}\right\}$ of $J$ is bounded in $E$.

Proof. We first prove (2.7). For $t>0$, write

$$
V=(V-t)_{+}+t-(V-t)_{-} .
$$

Then we have

$$
\begin{aligned}
& \int_{\mathbb{R}^{n}} V|u|^{m}=\int_{\mathbb{R}^{n}}\left[(V-t)_{+}+t\right]|u|^{m}-\int_{\mathbb{R}^{n}}(V-t)_{-}|u|^{m} \\
& \geq \int_{\mathbb{R}^{n}}\left[(V-t)_{+}+t\right]|u|^{m}-\left(\int_{\mathbb{R}^{n}}(V-t)_{-}^{n / m}\right)^{m / n}\left(\int_{\mathbb{R}^{n}}|u|^{m^{*}}\right)^{m / m^{*}} \\
&(2.8) \quad \geq \int_{\mathbb{R}^{n}}\left[(V-t)_{+}+t\right]|u|^{m}-S^{-1}\left(\int_{\mathbb{R}^{n}}(V-t)_{-}^{n / m}\right)^{m / n} \int_{\mathbb{R}^{n}}|\nabla u|^{m} .
\end{aligned}
$$

We claim that there exist $\varepsilon_{0}>0$ and $t_{0} \in\left(0, \tau_{0}\right)$ such that

$$
V_{t}=\int_{\mathbb{R}^{n}}(V-t)_{-}^{n / m}<S^{n / m}-\varepsilon_{0}, \quad t \in\left(0, t_{0}\right] .
$$

For simplicity, we restrict $t \in\left(0, \tau_{0}\right)$. For $R>0$, put

$$
D=D_{R, t}=\Omega_{t} \cap B_{R}(0), \quad T=T_{R, t}=\Omega_{t} \cap\left(\mathbb{R}^{n} \backslash B_{R}(0)\right) .
$$

Clearly $|T| \rightarrow 0$ as $R \rightarrow \infty$ uniformly in $t$ since $\Omega_{t} \subset \Omega_{\tau_{0}}$ has a finite volume. Set

$$
3 \varepsilon_{0}:=S^{n / m}-V_{0}^{n / m}>0 .
$$

Then there exists $R_{0}>0$ such that

$$
\int_{T}(V-t)_{-}^{n / m} \leq C \int_{T} V_{-}^{n / m}+C t|T|<\varepsilon_{0}, \quad R \geq R_{0}
$$

uniformly for all $t \in\left(0, \tau_{0}\right)$, since $V_{-} \in L^{n / m}\left(\mathbb{R}^{n}\right)$. Fixing $R=R_{0}$, one readily infers that there exists $t_{0}>0$ such that for $t \in\left(0, t_{0}\right]$,

$$
\int_{D}(V-t)_{-}^{n / m}<V_{0}^{n / m}+\varepsilon_{0}=S^{n / m}-2 \varepsilon_{0},
$$


since $(V-t)_{-}^{n / m} \rightarrow V_{-}^{n / m}$ uniformly on $\mathbb{R}^{n}$ and $D \rightarrow \Omega_{0} \cap B_{R_{0}}(0)$ as $t \rightarrow 0$. Combining (2.10) and (2.11) immediately yields (2.9).

It follows that, by substituting (2.9) into (2.8),

$$
\begin{aligned}
L(u) & =\int_{\mathbb{R}^{n}}\left[|\nabla u|^{m}+V|u|^{m}\right] \\
& \geq \int_{\mathbb{R}^{n}}|\nabla u|^{m}+\int_{\mathbb{R}^{n}}\left[\left(V-t_{0}\right)_{+}+t_{0}\right]|u|^{m}-S^{-1} V_{t_{0}}^{m / n} \int_{\mathbb{R}^{n}}|\nabla u|^{m} \\
& =\int_{\mathbb{R}^{n}}\left[\left(V-t_{0}\right)_{+}+t_{0}\right]|u|^{m}+\left(1-V_{t_{0}}^{m / n} S^{-1}\right) \int_{\mathbb{R}^{n}}|\nabla u|^{m} \\
& \geq t_{0} \int_{\mathbb{R}^{n}}|u|^{m}+\left(1-V_{t_{0}}^{m / n} S^{-1}\right) \int_{\mathbb{R}^{n}}|\nabla u|^{m} \geq \varepsilon_{1}\|u\|_{1}^{m},
\end{aligned}
$$

since $\left(V-t_{0}\right)_{+} \geq 0$, where

$$
\varepsilon_{1}=\min \left\{t_{0},\left(1-V_{t_{0}}^{m / n} S^{-1}\right)\right\}>0 .
$$

On the other hand, by definition

$$
\begin{aligned}
L(u) & =\|u\|^{m}-\int_{\mathbb{R}^{n}} V_{-}|u|^{m} \\
& \geq\|u\|^{m}-V_{0}\left(\int_{\mathbb{R}^{n}}|u|^{m^{*}}\right)^{m / m^{*}} \\
& \geq\|u\|^{m}-V_{0} S^{-1} \int_{\mathbb{R}^{n}}|\nabla u|^{m} \geq\left(1-V_{0} S^{-1}\right)\|u\|^{m} .
\end{aligned}
$$

Now (2.7) follows from (2.12)-(2.13) immediately since

$$
1-V_{0} S^{-1}>0, \quad\|u\|^{m} \geq L(u) .
$$

Next let $\left\{u_{j}\right\}$ be a (PS) sequence at level-c. Then

$$
J\left(u_{j}\right)=c+o(1), \quad J^{\prime}\left(u_{j}\right) u_{j}=o\left(\left\|u_{j}\right\|\right) .
$$

By assumption, there exists a positive number $\alpha_{1} \in\left(m, m^{*}\right)$ such that

$$
u f(x, u) \geq \alpha_{1} F(x, u) .
$$

It follows that, with the aid of (2.7),

$$
\begin{aligned}
\alpha_{1} c+o(1)+o\left(\left\|u_{j}\right\|\right)= & \alpha_{1} J\left(u_{j}\right)-J^{\prime}\left(u_{j}\right) u_{j} \\
= & \frac{\alpha_{1}-m}{m} L\left(u_{j}\right)+\left(m^{*}-\alpha_{1}\right) N\left(u_{j}\right) \\
& +\int_{\mathbb{R}^{n}}\left[u_{j} f\left(x, u_{j}\right)-\alpha_{1} F\left(x, u_{j}\right)\right] \\
\geq & \frac{\alpha_{1}-m}{m} L\left(u_{j}\right) \geq C\left\|u_{j}\right\|^{m} .
\end{aligned}
$$

The conclusion of the lemma follows immediately. 


\section{Convergence of (PS) sequences}

In this section, we prove the following convergence theorem.

Theorem 3.1. There exists a positive number $c_{0}=c_{0}\left(n, m, \alpha_{1}, \alpha_{2}, \beta, \tau_{0}, H_{0}\right)>$ 0 such that every (PS) sequence $\left\{u_{j}\right\}$ of $J$ at a critical-level $c$ converges in $L^{m^{*}}\left(\mathbb{R}^{n}\right)$, provided

$$
c<c_{0}
$$

Before proving Theorem 3.1, we establish some estimates in the following two technical lemmas. Let $\left\{u_{j}\right\}$ be a bounded (PS) sequence in $E$ and so Lemma 2.2 applies. We use the same notations from Section 2. We first give a lower bound for $\lambda_{i}$ values in (2.5).

Lemma 3.1. Assume $I \neq \emptyset$ in Lemma 2.2. Then there holds

$$
H\left(z_{i}\right)>0, \quad \lambda_{i} \geq\left(S / H\left(z_{i}\right)\right)^{n / m} \geq\left(S / H_{0}\right)^{n / m}, \quad i \in I .
$$

In particular, the set I is finite.

Proof. Let $\eta \in C^{\infty}([0, \infty))$ be a standard cut-off function on $[0,1]$, that is,

$$
\eta(t) \equiv 1, \quad t \in[0,1] ; \quad \eta(t) \equiv 0, \quad t>2 ; \quad\left|\eta^{\prime}(t)\right| \leq C, \quad 0 \leq \eta(t) \leq 1
$$

for some $C>0$. Fix $i \in I$. For $\varepsilon>0$, put

$$
\eta_{\varepsilon}=\eta\left(\left|x-z_{i}\right| / \varepsilon\right), \quad B=B_{2 \varepsilon}\left(z_{i}\right), \quad T=T_{\varepsilon}=B \backslash B_{\varepsilon}\left(z_{i}\right) .
$$

Apply $J^{\prime}\left(u_{j}\right)$ to the test function $u_{j} \eta_{\varepsilon}$ to obtain

$$
\begin{aligned}
J^{\prime}\left(u_{j}\right)\left(u_{j} \eta_{\varepsilon}\right)= & \int_{\mathbb{R}^{n}}\left|\nabla u_{j}\right|^{m} \eta_{\varepsilon}+\int_{\mathbb{R}^{n}} u_{j}\left|\nabla u_{j}\right|^{m-2} \nabla u_{j} \nabla \eta_{\varepsilon} \\
& +\int_{\mathbb{R}^{n}} V\left|u_{j}\right|^{m} \eta_{\varepsilon}-\int_{\mathbb{R}^{n}} f\left(x, u_{j}\right)\left(u_{j} \eta_{\varepsilon}\right)-\int_{\mathbb{R}^{n}} H\left|u_{j}\right|^{m^{*}} \eta_{\varepsilon} .
\end{aligned}
$$

We shall estimate the right hand-side of (3.3) term by term.

By definition, a direct computation yields

$$
\left\|u_{j} \eta_{\varepsilon}\right\|=\left(\int_{\mathbb{R}^{n}}\left(\left|\nabla\left(u_{j} \eta_{\varepsilon}\right)\right|^{m}+V^{0}\left|u_{j} \eta_{\varepsilon}\right|^{m}\right)\right)^{1 / m} \leq C\left\|u_{j}\right\|=O(1) .
$$

Hence

$$
J^{\prime}\left(u_{j}\right)\left(u_{j} \eta_{\varepsilon}\right)=o\left(\left\|u_{j} \eta_{\varepsilon}\right\|\right)=o(1)
$$

since $\left\{u_{j}\right\}$ is a (PS) sequence. By Lemma 2.2,

$$
\int_{\mathbb{R}^{n}}\left|\nabla u_{j}\right|^{m} \eta_{\varepsilon} \rightarrow \mu_{i}^{\prime} \geq \mu_{i}
$$

Clearly,

$$
\left|\nabla \eta_{\varepsilon}\right| \leq C / \varepsilon, \quad\left|\nabla \eta_{\varepsilon}\right|=0 \text { for } x \notin T, \quad \int_{T} 1=|T|=c_{n} \varepsilon^{n},
$$


where $|T|$ is the volume of $T$. Hence we apply the Hölder inequality and Lemma 2.2 to derive

$$
\begin{aligned}
& \left.\left|\int_{\mathbb{R}^{n}} u_{j}\right| \nabla u_{j}\right|^{m-2} \nabla u_{j} \nabla \eta_{\varepsilon} \mid \\
\leq & C \varepsilon^{-1} \int_{T}\left|\nabla u_{j}\right|^{m-1}\left|u_{j}\right| \\
\leq & C \varepsilon^{-1}\left(\int_{T} 1^{n}\right)^{1 / n}\left(\int_{T}\left|\nabla u_{j}\right|^{m}\right)^{(m-1) / m}\left(\int_{T}\left|u_{j}\right|^{m^{*}}\right)^{1 / m^{*}} \\
\leq & C \varepsilon^{-1}\left(\varepsilon^{n}\right)^{1 / n}\left(\int_{\mathbb{R}^{n}}\left|\nabla u_{j}\right|^{m}\right)^{(m-1) / m}\left(\int_{T}\left|u_{j}\right|^{m^{*}}\right)^{1 / m^{*}} \\
\leq & C\left(\sum_{i \in I^{\prime}} \lambda_{i}+\int_{T}\left|u_{0}\right|^{m^{*}}\right)^{1 / m^{*}}+o(1)=O(1),
\end{aligned}
$$

where $I^{\prime}=I_{\varepsilon}^{\prime}$ is the subset of $I$ satisfying

$$
\left\{z_{i} \mid i \in I^{\prime}\right\}=\left\{z_{i} \mid i \in I\right\} \cap T .
$$

We claim that

$$
\sum_{i \in I^{\prime}} \lambda_{i}=o(1) \quad \text { as } \varepsilon \rightarrow 0^{+} .
$$

This is trivial if $I$ is finite (then $I^{\prime}$ is empty for all small $\varepsilon>0$ ). If $I=Z$, then we assert that

$$
\lim _{\varepsilon \rightarrow 0^{+}} \min _{i \in I^{\prime}} i=\infty .
$$

Suppose the contrary, then there exists $i_{0}>0$ such that $i_{0} \in I^{\prime}$ for all $\varepsilon>0$. In turn, $z_{i_{0}} \in T$ for all $\varepsilon>0$. This is impossible in view of the definition of $T$. It follows that, since the series $\sum_{i \in I} \lambda_{i}$ converges, (3.6) necessarily holds (cf., the tail of a convergent series).

On the other hand, plainly, as $\varepsilon \rightarrow 0^{+}$,

$$
\int_{B}\left(\left|u_{0}\right|^{m^{*}}+\left|\nabla u_{0}\right|^{m}\right)=o(1) \text {. }
$$

Thus in fact

$$
\int_{\mathbb{R}^{n}} u_{j}\left|\nabla u_{j}\right|^{m-2} \nabla u_{j} \nabla \eta_{\varepsilon}=o(1) .
$$

Using the Hölder inequality, we have

$$
\left.\left|\int_{\mathbb{R}^{n}} V\right| u_{j}\right|^{m} \eta_{\varepsilon} \mid \leq\left(\int_{B}|V|^{n / m}\right)^{m / n}\left(\int_{B}\left|u_{j}\right|^{m^{*}}\right)^{m / m^{*}}=o(1) O\left(\| u_{j}||\right)=o(1),
$$

since $V \in L_{l o c}^{n / m}\left(\mathbb{R}^{n}\right)$. By the Hölder inequality again, for all $\sigma \in\left[0, m^{*}\right)$, one has

$$
\int_{B}\left|u_{j}\right|^{\sigma} \leq|B|^{1-\sigma / m^{*}}\left(\int_{\mathbb{R}^{n}}\left|u_{j}\right|^{m^{*}}\right)^{\sigma / m^{*}}=o(1) .
$$


Hence, by assumption, there holds

$$
\int_{\mathbb{R}^{n}} f\left(x, u_{j}\right)\left(u_{j} \eta_{\varepsilon}\right) \leq \beta \int_{\mathbb{R}^{n}}\left(\left|u_{j}\right|^{\alpha_{1}}+\left|u_{j}\right|^{\alpha_{2}}\right) \eta_{\varepsilon}=o(1),
$$

where $\alpha_{1}, \alpha_{2} \in\left(m, m^{*}\right)$. Using the continuity of $H(x)$ and Lemma 2.2, and passing to the limit by first letting $j \rightarrow \infty$ and then letting $\varepsilon \rightarrow 0_{+}$, we have

$$
\lim _{j \rightarrow \infty} \int_{\mathbb{R}^{n}} H\left|u_{j}\right|^{m^{*}} \eta_{\varepsilon}=H\left(z_{i}\right) \lambda_{i} .
$$

Combining (3.4)-(3.5) and (3.7)-(3.10) into (3.3), we infer that for each fixed $i \in I$

$$
\mu_{i}-H\left(z_{i}\right) \lambda_{i} \leq 0
$$

Utilizing (2.6), we finally arrive at

$$
S \lambda_{i}^{m / m^{*}}-H\left(z_{i}\right) \lambda_{i} \leq 0 .
$$

Clearly $H\left(z_{i}\right)>0$, since $\lambda_{i}>0$. Thus (3.2) follows and the proof is complete.

For $R>0$, put

$$
\eta_{R}=\eta(2 R /|x|), \quad D=D_{R}=\mathbb{R}^{n} \backslash B_{R}(0), \quad T=T_{R}=B_{2 R}(0) \backslash B_{R}(0) .
$$

Denote

$$
\begin{gathered}
\mu_{\infty}=\lim _{R \rightarrow \infty} \lim _{j \rightarrow \infty} \int_{\mathbb{R}^{n}}\left|\nabla u_{j}\right|^{m} \eta_{R}, \quad V_{\infty}=\lim _{R \rightarrow \infty} \lim _{j \rightarrow \infty} \int_{\mathbb{R}^{n}} V\left|u_{j}\right|^{m} \eta_{R}, \\
F_{\infty}=\lim _{R \rightarrow \infty} \lim _{j \rightarrow \infty} \int_{\mathbb{R}^{n}} u_{j} f\left(x, u_{j}\right) \eta_{R},
\end{gathered}
$$

and

$$
\lambda_{\infty}=\lim _{R \rightarrow \infty} \lim _{j \rightarrow \infty} \int_{\mathbb{R}^{n}}\left|u_{j}\right|^{m^{*}} \eta_{R}, \quad \lambda_{\infty}^{\prime}=\lim _{R \rightarrow \infty} \lim _{j \rightarrow \infty} \int_{\mathbb{R}^{n}} H\left|u_{j}\right|^{m^{*}} \eta_{R} .
$$

Note that all five limits are non-negative. For $k=1,2$, put

$$
a_{k}=\frac{m^{*}-\alpha_{k}}{m^{*}-m} \in(0,1), \quad b_{k}=\frac{\alpha_{k}-m}{m^{*}-m} \in(0,1) ; \quad a_{k}+b_{k}=1 .
$$

Then we have the following estimates.

Lemma 3.2. There holds

$$
\mu_{\infty}+V_{\infty} \leq H_{0} \lambda_{\infty}+\beta \sum_{k=1}^{2}\left(V_{\infty} / \tau_{0}\right)^{a_{k}} \lambda_{\infty}^{b_{k}} .
$$

If in addition $\lambda_{\infty}>0$, then there exists $C=C\left(n, m, \alpha_{1}, \alpha_{2}, \beta, \tau_{0}, H_{0}\right)>0$ such that

$$
\mu_{\infty} \geq S \lambda_{\infty}^{m / m^{*}} \geq C
$$


Proof. The proof is essentially the same as that of Lemma 3.1. Applying $J^{\prime}\left(u_{j}\right)$ to the test function $u_{j} \eta_{R}$, we obtain

$$
\mu_{\infty}+V_{\infty}=\lambda_{\infty}^{\prime}+F_{\infty} .
$$

To show (3.11), we shall bound $F_{\infty}$ in terms of $V_{\infty}$ and $\lambda_{\infty}$.

By the Hölder inequality, we have

$$
\int_{\mathbb{R}^{n}}\left(V-\tau_{0}\right)_{-}\left|u_{j}\right|^{m} \eta_{R} \leq\left(\int_{D}\left(V-\tau_{0}\right)_{-}^{n / m}\right)^{m / n}\left(\int_{\mathbb{R}^{n}}\left|u_{j}\right|^{m^{*}}\right)^{(n-m) / n}=o(1),
$$

since $\left(V-\tau_{0}\right)_{-} \in L^{n / m}\left(\mathbb{R}^{n}\right)$. In turn

$$
\begin{aligned}
\int_{\mathbb{R}^{n}} V\left|u_{j}\right|^{m} \eta_{R} & =\int_{\mathbb{R}^{n}}\left[\left(V-\tau_{0}\right)_{+}+\tau_{0}\right]\left|u_{j}\right|^{m} \eta_{R}-\int_{\mathbb{R}^{n}}\left(V-\tau_{0}\right)_{-}\left|u_{j}\right|^{m} \eta_{R} \\
& =\int_{\mathbb{R}^{n}}\left[\left(V-\tau_{0}\right)_{+}+\tau_{0}\right]\left|u_{j}\right|^{m} \eta_{R}+o(1) .
\end{aligned}
$$

It follows that

$$
V_{\infty}=\lim _{R \rightarrow \infty} \lim _{j \rightarrow \infty} \int_{\mathbb{R}^{n}} V(x)\left|u_{j}\right|^{m} \eta_{R} \geq \tau_{0} \lim _{R \rightarrow \infty} \lim _{j \rightarrow \infty} \int_{\mathbb{R}^{n}}\left|u_{j}\right|^{m} \eta_{R} .
$$

By assumption (F), there holds

$$
0 \leq f\left(x, u_{j}\right) u_{j} \leq \beta\left(\left|u_{j}\right|^{\alpha_{1}}+\left|u_{j}\right|^{\alpha_{2}}\right),
$$

where $\alpha_{1}, \alpha_{2} \in\left(m, m^{*}\right)$. It follows that, with the aid of the Hölder inequality

$$
\begin{aligned}
\int_{\mathbb{R}^{n}} f\left(x, u_{j}\right)\left(u_{j} \eta_{R}\right) & \leq \beta \int_{\mathbb{R}^{n}}\left(\left|u_{j}\right|^{\alpha_{1}}+\left|u_{j}\right|^{\alpha_{2}}\right) \eta_{R} \\
& \leq \beta \sum_{k=1}^{2}\left(\int_{\mathbb{R}^{n}}\left|u_{j}\right|^{m} \eta_{R}\right)^{a_{k}}\left(\int_{\mathbb{R}^{n}}\left|u_{j}\right|^{m^{*}} \eta_{R}\right)^{b_{k}} .
\end{aligned}
$$

Combining (3.14) and (3.15) yields

$$
F_{\infty} \leq \beta \sum_{k=1}^{2}\left(V_{\infty} / \tau_{0}\right)^{a_{k}} \lambda_{\infty}^{b_{k}} .
$$

Plainly

$$
\lambda_{\infty}^{\prime} \leq H_{0} \lim _{R \rightarrow \infty} \lim _{j \rightarrow \infty} \int_{\mathbb{R}^{n}}\left|u_{j}\right|^{m^{*}} \eta_{R}=H_{0} \lambda_{\infty} .
$$

Substituting (3.16) and (3.17) into (3.13) yields (3.11).

To prove (3.12), we apply the Sobolev embedding to $u_{j} \eta_{R}$ to obtain

$$
\begin{aligned}
S \lambda_{\infty}^{m / m^{*}} & =S \lim _{R \rightarrow \infty} \lim _{j \rightarrow \infty}\left(\int_{\mathbb{R}^{n}}\left|u_{j} \eta_{R}\right|^{m^{*}}\right)^{m / m^{*}} \\
& \leq \lim _{R \rightarrow \infty} \lim _{j \rightarrow \infty} \int_{\mathbb{R}^{n}}\left|\nabla\left(u_{j} \eta_{R}\right)\right|^{m}=\mu_{\infty} .
\end{aligned}
$$


This is (3.12) $)_{1}$. Using the above inequality, (3.12) $)_{2}$ follows from (3.11) and the Young inequality with the aid of the fact $a_{k} \in(0,1)$, and $a_{k}+b_{k}=1$, $k=1,2$.

Now we are ready to prove Theorem 3.1.

Proof of Theorem 3.1. Let $\left\{u_{j}\right\}$ be a (PS) sequence of $J$ at level-c. By Lemma $2.3,\left\{u_{j}\right\}$ is bounded in $E$. Hence, without loss of generality, we may assume that $\left\{u_{j}\right\}$ converges weakly and a.e. to some $u_{0} \in E$. We want to show that the singular part and escaping part of the limiting energy-level of the sequence $\left\{u_{j}\right\}$ are trivial, provided (3.1) is satisfied. This would in turn ensure the convergence of $\left\{u_{j}\right\}$ in $L^{m^{*}}\left(\mathbb{R}^{n}\right)$.

We first claim

$$
\lambda_{\infty}=\mu_{\infty}=F_{\infty}=V_{\infty}=0, \quad I=\emptyset .
$$

By the compact Sobolev embedding, we have for any fixed $R>0$ and $\alpha \in$ $\left(0, m^{*}\right)$

$$
\lim _{j \rightarrow \infty} \int_{B}\left|u_{j}\right|^{\alpha}=\int_{B}\left|u_{0}\right|^{\alpha},
$$

where $B$ is ball with radius $R$. Hence

$$
\lim _{j \rightarrow \infty} \int_{\mathbb{R}^{n}} u_{j} f\left(x, u_{j}\right)=\int_{\mathbb{R}^{n}} u_{0} f\left(x, u_{0}\right)+F_{\infty} .
$$

By Lemma 2.2

$$
\lim _{j \rightarrow \infty} \int_{\mathbb{R}^{n}} H\left|u_{j}\right|^{m^{*}}=\lambda_{\infty}^{\prime}+\sum_{i \in I} H\left(z_{i}\right) \lambda_{i}+\int_{\mathbb{R}^{n}} H\left|u_{0}\right|^{m^{*}} .
$$

It follows that

$$
\begin{aligned}
c+o(1)= & J\left(u_{j}\right)-\frac{1}{m} J^{\prime}\left(u_{j}\right) u_{j} \\
= & \frac{1}{m} \int_{\mathbb{R}^{n}}\left[u_{j} f\left(x, u_{j}\right)-m F\left(x, u_{j}\right)\right]+m^{\prime} \int_{\mathbb{R}^{n}} H\left|u_{j}\right|^{m^{*}} \\
\geq & \alpha^{\prime} \int_{\mathbb{R}^{n}} u_{j} f\left(x, u_{j}\right)+m^{\prime} \int_{\mathbb{R}^{n}} H\left|u_{j}\right|^{m^{*}} \\
= & \alpha^{\prime} \int_{\mathbb{R}^{n}} u_{0} f\left(x, u_{0}\right)+m^{\prime} \int_{\mathbb{R}^{n}} H\left|u_{0}\right|^{m^{*}} \\
& +m^{\prime}\left(\lambda_{\infty}^{\prime}+\sum_{i \in I} H\left(z_{i}\right) \lambda_{i}\right)+\alpha^{\prime} F_{\infty}+o(1) \\
\geq & m^{\prime} \sum_{i \in I} H\left(z_{i}\right) \lambda_{i}+\alpha^{\prime}\left(\lambda_{\infty}^{\prime}+F_{\infty}\right)+o(1) \\
\geq & m^{\prime} \sum_{i \in I} H\left(z_{i}\right) \lambda_{i}+\alpha^{\prime} \mu_{\infty}+o(1)
\end{aligned}
$$


where we have used the fact $\lambda_{\infty}^{\prime}+F_{\infty}=\mu_{\infty}+V_{\infty} \geq \mu_{\infty}$, and

$$
m^{\prime}=\frac{m^{*}-m}{m m^{*}}>\alpha^{\prime}=\frac{\alpha_{1}-m}{m \alpha_{1}}>0 .
$$

Take

$$
c_{0}=\min \left\{\alpha^{\prime} C, m^{\prime} S^{n / m} H_{0}^{(m-n) / m}\right\}>0,
$$

where the constant $C>0$ is given in (3.12). Suppose the contrary $\lambda_{\infty}>0$, then one has, by (3.2) and (3.19),

$$
c \geq \alpha^{\prime} \mu_{\infty} \geq \alpha^{\prime} C=c_{0},
$$

a contradiction to the assumption $c<c_{0}$. Hence $\lambda_{\infty}=0$. Using this fact, one immediately infers $\mu_{\infty}=V_{\infty}=0$ by (3.11) and $F_{\infty}=0$ by (3.16), respectively. Similarly, one deduces $I=\emptyset$ using (3.2), (3.19) and the definition of $c_{0}$.

Now with (3.18) available, one uses Lemma 2.2 to derive

$$
\lim _{j \rightarrow \infty} \int_{\mathbb{R}^{n}}\left|u_{j}\right|^{m^{*}}=\int_{\mathbb{R}^{n}}\left|u_{0}\right|^{m^{*}} .
$$

In turn (see e.g. [4])

$$
\lim _{j \rightarrow \infty} \int_{\mathbb{R}^{n}}\left|u_{j}-u_{0}\right|^{m^{*}} \rightarrow 0,
$$

since $u_{j} \rightarrow u_{0}$ a.e. on $\mathbb{R}^{n}$. The proof is complete.

\section{The existence results}

In this section, we use the mountain-pass Lemma 2.1 and the convergence Theorem 3.1 to prove the existence Theorem 1.1. We also present three corollaries of Theorem 1.1.

Proof of Theorem 1.1. By definition and assumptions, there holds

$$
\begin{aligned}
J(u) & \geq \frac{1}{m} L(u)-\beta \int_{\mathbb{R}^{n}}\left(|u|^{\alpha_{1}}+|u|^{\alpha_{2}}\right)-\frac{H_{0}}{m^{*}} \int_{\mathbb{R}^{n}}|u|^{m^{*}} \\
& \geq 2 d\|u\|^{m}+2 d\|u\|_{1}^{m}-2 d \int_{\mathbb{R}^{n}}|u|^{m}-C \int_{\mathbb{R}^{n}}|u|^{m^{*}} \geq 2 d\|u\|^{m}-\left.C\|u\|\right|^{m^{*}},
\end{aligned}
$$

where $d=d\left(m, d_{0}, d_{1}\right)>0$ and we have used (2.7) and the Young inequality

$$
|u|^{\alpha_{k}} \leq d|u|^{m}+C_{k}|u|^{m^{*}}, \quad k=1,2 .
$$

Let $r_{0}>0$ be the positive solution of

$$
2 d r_{0}^{m}-C r_{0}^{m^{*}}=d r_{0}^{m} .
$$

Now taking $\rho=d r_{0}^{m}>0$ and $U$ the ball centered at the origin with radius $r_{0}$ verifies (2.1).

Take $\kappa_{0}=c_{0}$, where $c_{0}$ is the quantity given in Theorem 3.1. By assumption, there exists $\phi_{0} \in E$ with $\phi_{0} \neq 0$ such that

$$
\max _{t \geq 0} J\left(t \phi_{0}\right)<\kappa_{0}=c_{0}, \quad \liminf _{t \rightarrow \infty} J\left(t \phi_{0}\right)<0 .
$$


Plainly, there exists $t_{0}>0$ such that

$$
J\left(t_{0} \phi_{0}\right) \leq 0 \text { with }\left\|t_{0} \phi_{0}\right\|>r_{0} .
$$

Set

$$
v(x)=t_{0} \phi_{0}(x)
$$

Then $v(x)$ together with $J$ satisfies the requirements of Lemma 2.1 and the inequality

$$
c=c_{v}<c_{0} .
$$

Let $\left\{u_{j}\right\}$ be a (PS) sequence at level-c guaranteed by Lemma 2.1. Then by Lemma 2.3, $\left\{u_{j}\right\}$ is bounded and converges weakly and a.e. to some $u_{0} \in E$. Using a test function in $C_{0}^{\infty}\left(\mathbb{R}^{n}\right)$, one passes the limit in $J^{\prime}\left(u_{j}\right) \rightarrow 0$ to conclude that $u_{0}$ is a (weak) solution of (1.1). In particular,

$$
\int_{\mathbb{R}^{n}}\left|\nabla u_{0}\right|^{m}=\int_{\mathbb{R}^{n}}\left[u_{0} f\left(x, u_{0}\right)+H\left|u_{0}\right|^{m^{*}}-V\left|u_{0}\right|^{m}\right] .
$$

By Theorem 3.1, with the aid of (4.1), we have

$$
\lim _{j \rightarrow \infty} \int_{\mathbb{R}^{n}}\left|u_{j}-u_{0}\right|^{m^{*}}=0 .
$$

Moreover, Theorem 3.1 implies

$$
\lambda_{\infty}=\mu_{\infty}=F_{\infty}=V_{\infty}=0, \quad I=\emptyset .
$$

In turn

$$
\lim _{j \rightarrow \infty} \int_{\mathbb{R}^{n}} V\left|u_{j}-u_{0}\right|^{m}=0, \quad \lim _{j \rightarrow \infty} \int_{\mathbb{R}^{n}} u_{j} f\left(x, u_{j}\right)=\int_{\mathbb{R}^{n}} u_{0} f\left(x, u_{0}\right) .
$$

It follows that

$$
\begin{aligned}
0 & =\lim _{j \rightarrow \infty} J^{\prime}\left(u_{j}\right) u_{j} \\
& =\lim _{j \rightarrow \infty} \int_{\mathbb{R}^{n}}\left|\nabla u_{j}\right|^{m}+\lim _{j \rightarrow \infty} \int_{\mathbb{R}^{n}}\left(V\left|u_{j}\right|^{m}-u_{j} f\left(x, u_{j}\right)-H\left|u_{j}\right|^{m^{*}}\right) \\
& =\lim _{j \rightarrow \infty} \int_{\mathbb{R}^{n}}\left|\nabla u_{j}\right|^{m}+\int_{\mathbb{R}^{n}}\left[V\left|u_{0}\right|^{m}-u_{0} f\left(x, u_{0}\right)-H\left|u_{0}\right|^{m^{*}}\right] .
\end{aligned}
$$

That is, with the aid of (4.2),

$$
\lim _{j \rightarrow \infty} \int_{\mathbb{R}^{n}}\left|\nabla u_{j}\right|^{m}=\int_{\mathbb{R}^{n}}\left|\nabla u_{0}\right|^{m} .
$$

Therefore

$$
J\left(u_{0}\right)=\lim _{j \rightarrow \infty} J\left(u_{j}\right)=c>0,
$$

in view of (2.1) with $\rho=d r_{0}^{m}>0$. Hence $u_{0} \neq 0$.

We claim $u_{0}>0$. Recall that all the results above apply to the modified functional $J_{1}$. In turn, $u_{0}$ can be simply seen a critical point of $J_{1}$ (i.e., replacing 
$J$ by $J_{1}$ if one will). Thus, applying $J_{1}^{\prime}\left(u_{0}\right)$ to $u_{0}^{-}$yields (noting that modified 'non-linear' parts of $J_{1}$ vanish on the support of $u_{0}^{-}$)

$$
0=J_{1}^{\prime}\left(u_{0}\right) u_{0}^{-}=L\left(u_{0}^{-}\right)=\int_{\mathbb{R}^{n}}\left(\left|\nabla u_{0}^{-}\right|^{m}+V\left|\nabla u_{0}^{-}\right|^{m}\right) \geq\left\|u_{0}^{-}\right\|^{m},
$$

where $u_{0}^{-}$denotes the negative part of $u_{0}$. It follows that $u_{0} \geq 0$. Now the strong maximum principle implies $u_{0}>0$. This completes the proof.

Next we present several sufficient conditions to ensure the validity of the key assumption (1.5) of Theorem 1.1, which in turn gives rise to existence. The presence of the lower order term $f(x, u)$ as well as the behavior of the potential $V(x)$ are crucial to the arguments. In the remaining of this section, we assume that there exists $\alpha_{3} \in\left(m, m^{*}\right)$ such that

$$
F(x, u) \geq|u|^{\alpha_{3}} / \beta, \quad(x, u) \in \mathbb{R}^{n+1} .
$$

For convenience, put

$$
I(u):=\frac{1}{m} L(u)-\frac{1}{\alpha_{3}} M(u), \quad M(u):=\frac{\alpha_{3}}{\beta} \int_{\mathbb{R}^{n}}|u|^{\alpha_{3}} .
$$

Clearly

$$
J(u) \leq I(u)
$$

Set

$$
\delta=\frac{\alpha_{3}}{\alpha_{3}-m}>0, \quad \gamma=\frac{m}{\alpha_{3}-m}>0, \quad \sigma=n \gamma-(n-m) \delta>0 .
$$

For $u \neq 0$, by direct computations

$$
\max _{t \geq 0} I(t u)=\frac{1}{m \delta} L^{\delta}(u) M^{-\gamma}(u) .
$$

The following existence is an immediate corollary of Theorem 1.1.

Corollary 4.1. Suppose that the conditions of Theorem 1.1 are valid. Then there exists a positive number $R_{0}=R_{0}\left(n, m, \alpha_{1}, \alpha_{2}, \beta, \alpha_{3}, \tau_{0}, H_{0}\right)$ such that (1.1) admits a positive solution, provided

$$
L\left(\xi_{R_{1}}\right)=\int_{\mathbb{R}^{n}}\left[\left|\nabla \xi_{R_{1}}\right|^{m}+V \xi_{R_{1}}^{m}\right] \leq\left(R_{1} / R_{0}\right)^{\sigma / \delta} \int_{\mathbb{R}^{n}}\left|\nabla \xi_{R_{1}}\right|^{m}
$$

for some $R_{1} \geq R_{0}$, where $\xi_{R}=\eta\left(|x| R^{-1}\right.$ ) for $R>0$ (recall the definition of $\eta$ from Section 3$)$.

Proof. The idea is to construct a non-negative function $\phi_{0}$ so that the pair $\left(\phi_{0}, c_{0}\right)$ satisfy $(1.5)$, where $c_{0}=c_{0}\left(n, m, \alpha_{1}, \alpha_{2}, \beta, \tau_{0}, H_{0}\right)$ is given in Theorem 3.1.

For $R>0$, put

$$
g(R)=\frac{1}{m \delta}\left(\int_{\mathbb{R}^{n}}\left|\nabla \xi_{R}\right|^{m}\right)^{\delta} M^{-\gamma}\left(\xi_{R}\right) .
$$


Direct computations yield

$$
\int_{\mathbb{R}^{n}}\left|\nabla \xi_{R}\right|^{m} \leq C R^{n-m}, \quad M\left(\xi_{R}\right)=C R^{n},
$$

where $C=C(n)>0$ is constant. It follows that there exists $C=C(n, m, \delta, \gamma)$ $>0$ such that

$$
g(R) \leq C R^{-\sigma}
$$

Moreover, a simple calculation reveals for $R_{2}, R_{1}>0$

$$
R_{2}^{\sigma} g\left(R_{2}\right)=R_{1}^{\sigma} g\left(R_{1}\right) \text {. }
$$

Take

$$
R_{0}=\left(C c_{0}^{-1}\right)^{1 / \sigma},
$$

where the constant $C>0$ is given in (4.6). Let $R_{1} \geq R_{0}$ be the constant given in the assumption. Then we have, with the aid of (4.4)-(4.8),

$$
\begin{aligned}
\max _{t \geq 0} J\left(t \xi_{R_{1}}\right) & \leq \max _{t \geq 0} I\left(t \xi_{R_{1}}\right)=\frac{1}{m \delta} L^{\delta}\left(\xi_{R_{1}}\right) M^{-\gamma}\left(\xi_{R_{1}}\right) \\
& \leq \frac{\left(R_{1} / R_{0}\right)^{\sigma}}{m \delta}\left(\int_{\mathbb{R}^{n}}\left|\nabla \xi_{R_{1}}\right|^{m}\right)^{\delta} M^{-\gamma}\left(\xi_{R_{1}}\right) \\
& =\left(R_{1} / R_{0}\right)^{\sigma} g\left(R_{1}\right)=g\left(R_{0}\right) \leq C R_{0}^{-\sigma}=c_{0} .
\end{aligned}
$$

On the other hand, there holds

$$
\lim _{t \rightarrow \infty} J\left(t \xi_{R_{1}}\right) \leq \lim _{t \rightarrow \infty} I\left(t \xi_{R_{1}}\right)=\lim _{t \rightarrow \infty}\left(\frac{t^{m}}{m} \int_{\mathbb{R}^{n}}\left|\nabla \xi_{R_{1}}\right|^{m}-\frac{t^{\alpha_{3}}}{\beta} \int_{\mathbb{R}^{n}} \xi_{R_{1}}^{\alpha_{3}}\right)=-\infty,
$$

since $\alpha_{3}>m$. Evidently the pair $\left(\phi_{0}, \kappa_{0}\right)=\left(\xi_{R_{1}}, c_{0}\right)$ satisfies (1.5) of Theorem 1.1. Hence Theorem 1.1 applies and (1.1) has a positive solution.

The next result is a direct generalization of Theorem 1.1 of [6]. Consider

$$
\begin{aligned}
& -\varepsilon \Delta_{m} u+V(x) u^{m-1}-f(x, u)-H(x) u^{m^{*}-1}=0 \text { in } \mathbb{R}^{n} \\
& u \rightarrow 0 \quad \text { as }|x| \rightarrow \infty \text {, }
\end{aligned}
$$

where $\varepsilon>0$ is a parameter.

Corollary 4.2. Suppose that the conditions of Theorem 1.1 are valid. Assume that there exists $x_{0} \in \mathbb{R}^{n}$ such that $V$ is continuous at $x_{0}$ and

$$
V\left(x_{0}\right) \leq 0 \text {. }
$$

Then there exists a positive number $\varepsilon_{0}=\varepsilon_{0}\left(n, m, \alpha_{1}, \alpha_{2}, \beta, \alpha_{3}, \tau_{0}, H_{0}, V\right)$ such that (4.9) admits a positive solution for all $\varepsilon<\varepsilon_{0}$.

Proof. The proof is essentially the same as that of Corollary 4.1. Namely, we want to construct $\phi_{0}$ so that the pair $\left(\phi_{0}, \kappa_{0}\right)$ verifies (1.5). Without loss of generality, assume $x_{0}=0$. Then for $\sigma>0$, there exists a positive number $\rho=\rho(\sigma)>0$ such that

$$
V(x)<\sigma+V(0) \leq \sigma, \quad|x|<2 \rho .
$$


Denote

$$
\phi_{\rho}=\eta(|x| / \rho), \quad V_{\rho}=\sup _{|x|<2 \rho} V(x) .
$$

Then there exists $C=C(n)>0$ such that

$$
\int_{\mathbb{R}^{n}}\left|\nabla \phi_{\rho}\right|^{m}=C \rho^{n-m}, \quad \int_{\mathbb{R}^{n}} V\left|\phi_{\rho}\right|^{m} \leq C V_{\rho} \rho^{n},
$$

and

$$
M\left(\phi_{\rho}\right)=\frac{\alpha_{3}}{\beta} \int_{\mathbb{R}^{n}}\left|\phi_{\rho}\right|^{\alpha_{3}}=\frac{C \alpha_{3}}{\beta} \rho^{n} .
$$

It follows that

$$
\frac{1}{m \delta}\left(\varepsilon \int_{\mathbb{R}^{n}}\left|\nabla \phi_{\rho}\right|^{m}+\int_{\mathbb{R}^{n}} V\left|\phi_{\rho}\right|^{m}\right)^{\delta} M^{-\gamma}\left(\phi_{\rho}\right) \leq C\left(\varepsilon \rho^{-m}+V_{\rho}\right)
$$

for some $C=C\left(n, m, \beta, \alpha_{3}\right)>0$. Choose

$$
\sigma_{0}=\frac{1}{2} c_{0} / C>0
$$

where $c_{0}=c_{0}\left(n, m, \alpha_{1}, \alpha_{2}, \beta, \tau_{0}, H_{0}\right)$ is given in Theorem 3.1. Fix $\rho_{0}>0$ such that (4.8) holds with $\sigma=\sigma_{0}$ and $\rho=\rho_{0}$. Now take

$$
\varepsilon_{0}=\frac{1}{2} c_{0} \rho_{0}^{m} / C>0 \quad \Longrightarrow \quad C\left(\varepsilon_{0} \rho_{0}^{-m}+\sigma_{0}\right)=c_{0} .
$$

Note that (4.4) remains valid for (4.7) with

$$
L(u)=\varepsilon \int_{\mathbb{R}^{n}}|\nabla u|^{m}+\int_{\mathbb{R}^{n}} V|u|^{m} .
$$

Hence for $\varepsilon<\varepsilon_{0}$, one infers that,

$$
\begin{aligned}
\max _{t \geq 0} I\left(t \phi_{\rho_{0}}\right) & \leq \max _{t \geq 0} I\left(t \phi_{\rho_{0}}\right)=\frac{1}{m \delta} L^{\delta}\left(\phi_{\rho_{0}}\right) M^{-\gamma}\left(\phi_{\rho_{0}}\right) \\
& \leq C\left(\varepsilon \rho_{0}^{-m}+V_{\rho_{0}}\right)<C\left(\varepsilon_{0} \rho_{0}^{-m}+\sigma_{0}\right)=c_{0},
\end{aligned}
$$

since $V_{\rho_{0}} \leq \sigma_{0}$ by (4.8). Now the pair $\left(\phi_{0}, \kappa_{0}\right)=\left(\phi_{\rho_{0}}, c_{0}\right)$ verifies $(1.5)$ and the proof is complete by Theorem 1.1 .

As mentioned earlier in the Introduction, the lower order perturbation term $f(x, u)$ plays a key role in the existence results. Indeed, Theorem 5.2 in Section 5, which shows that (1.1) has no positive solutions in general when $f(x, u) \equiv 0$, only enforces this notion. Corollary 4.3 below, however, indicates that (1.1) may still have positive solutions even with $f(x, u) \equiv 0$. In turn, this illustrates the critical importance of the behavior of the potential function $V(x)$. Also note that, in Corollary 4.3, the potential function $V(x)$ need not satisfy the condition (V), which is required for all Theorems 1.1 and 3.1, and Corollaries 4.1 and 4.2. 
Corollary 4.3. Assume $f \equiv 0$ and $H \equiv 1$. Suppose that $V \in L^{n / m}\left(\mathbb{R}^{n}\right) \cap$ $L_{\text {loc }}^{\infty}\left(\mathbb{R}^{n}\right)$ satisfies

where

$$
V_{0}=\left(\int_{\mathbb{R}^{n}} V_{-}^{n / m}\right)^{m / n}<S, \quad \int_{\mathbb{R}^{n}} V \phi^{m}<0,
$$

$$
\phi(x)=\left(\kappa+|x|^{m /(m-1)}\right)^{(m-n) / m}, \quad \int_{\mathbb{R}^{n}} \phi^{m^{*}}=1, \quad \int_{\mathbb{R}^{n}}|\nabla \phi|^{m}=S .
$$

Then (1.1) admits a positive solution.

Proof. The proof is essentially the same as that of Theorem 1.1, with the exception that one should work on the space $\mathcal{D}^{1, m}\left(\mathbb{R}^{n}\right)$. Namely, it is the completion of all compactly supported smooth functions on $\mathbb{R}^{n}$ under the Dirichlet norm

$$
\|u\|:=\left(\int_{\mathbb{R}^{n}}|\nabla u|^{m}\right)^{1 / m} .
$$

Then the previous arguments carry over with little change (see $[7,8]$ for the concentration-compactness principle for the space $\left.\mathcal{D}^{1, m}\left(\mathbb{R}^{n}\right)\right)$. Indeed, arguments of Lemma 3.1 directly imply

$$
\min _{i}\left\{\lambda_{i}\right\} \geq S^{n / m}
$$

provided $I \neq \emptyset$. On the other hand, to apply Lemma 3.2, we note that

$$
F_{\infty}=0, \quad \lambda_{\infty}^{\prime}=\lambda_{\infty}, \quad H_{0}=1
$$

Thus, one has

$$
\mu_{\infty}+V_{\infty}=\lambda_{\infty}, \quad \mu_{\infty} \geq S \lambda_{\infty}^{m / m^{*}} .
$$

By definition, one readily sees

$$
\begin{aligned}
\left|V_{\infty}\right| & =\left.\lim _{R \rightarrow \infty} \lim _{j \rightarrow \infty}\left|\int_{\mathbb{R}^{n}} V\right| u_{j} \eta_{R}\right|^{m} \mid \\
& \leq \lim _{R \rightarrow \infty} \lim _{j \rightarrow \infty}\left(\int_{|x|>R}|V|^{n / m}\right)^{m / n}\left(\int_{\mathbb{R}^{n}}\left|u_{j}\right|^{m^{*}}\right)^{m / m^{*}}=0 .
\end{aligned}
$$

Combining (4.12) and (4.13) yields

$$
\lambda_{\infty} \geq S^{n / m}
$$

provided $\lambda_{\infty}>0$. Using the assumption, direct computations show

$$
\max _{t \geq 0} J(t \phi)=\frac{1}{n} L^{n / m}(\phi)<\frac{1}{n}\left(\int_{\mathbb{R}^{n}}|\nabla \phi|^{m}\right)^{n / m}=\frac{S^{n / m}}{n} .
$$

Now choose

$$
c_{0}=m^{\prime} S^{n / m}=\frac{S^{n / m}}{n} .
$$

Then Theorem 3.1 applies and the pair $\left(\phi_{0}, \kappa_{0}\right)=\left(\phi, c_{0}\right)$ verifies $(1.5)$. Thus the conclusion follows from Theorem 1.1 and the details are left to the interested reader. 


\section{The non-existence results}

All results established in the previous section indeed show that both the presence of the lower order term $f$ and the behavior of the potential $V(x)$ are crucial for the existence. To further enforce such a notion, here we present three non-existence results. We shall assume none of the conditions $(\mathrm{V}),(\mathrm{F})$ and $(\mathrm{H})$. For simplicity, we shall also assume all solutions treated in this section are $C^{1}$.

The first non-existence result is when $V(x)$ becomes non-positive for large $|x|$ 's (and the lower order perturbation $f(x, u)$ has a low exponent).

Theorem 5.1. Assume that there exist positive numbers $C>0, R>0$ and

$$
m-1 \leq \alpha \leq \frac{(m-1) n}{n-m}
$$

such that there holds for $u>0$ sufficiently small and $|x|>R$,

$$
-V(x) u^{m-1}+f(x, u) \geq C u^{\alpha} .
$$

Then (1.1) does not admit any non-negative non-trivial solutions.

Theorem 5.1 is due to [3] (see also [12]), in view of the boundary conditions

$$
\lim _{|x| \rightarrow \infty} u(x)=0 .
$$

Theorem 5.2 below is a non-existence result when $f(x, u) \equiv 0$.

Theorem 5.2. Assume that the functions $f(x, u), V(x)$ and $H(x)$ satisfy the following conditions.

A) $H(x) \in C^{1}\left(\mathbb{R}^{n}\right)$ and there holds

$$
f(x, u) \equiv 0, \quad \sup _{x \in \mathbb{R}^{n}} x \cdot \nabla H(x) \leq 0, \quad \sup _{x \in \mathbb{R}^{n}}|H(x)|<\infty .
$$

B) There exist positive constants $R_{0}>0$ and $K_{0}>0$ such that

$$
K_{0}^{-1} \leq V(x) \leq K_{0} \quad \text { for } \quad|x|>R_{0}
$$

C) $C(x) \in C^{1}\left(\mathbb{R}^{n}\right)$ and there holds

$$
\left(|x|^{m} V(x)\right)^{\prime}>0 \quad \text { in } \quad \mathbb{R}^{n}
$$

where' is the derivative with respect to the radius $|x|$.

Then (1.1) does not admit any non-negative non-trivial solutions $u \in E$.

The next non-existence, however, reflects the importance of the behavior of the potential $V$ on existence, even with the presence of $f$.

Theorem 5.3. Assume that the functions $f(x, u), V(x)$ and $H(x)$ satisfy the following conditions.

A) $H(x) \in C^{1}\left(\mathbb{R}^{n}\right)$ and there holds

$$
\sup _{x \in \mathbb{R}^{n}} x \cdot \nabla H(x) \geq 0 ; \quad H=O(1) \text { and } \nabla H=O(1) \text { as }|x| \rightarrow \infty .
$$


B) $C(x) \in C^{1}\left(\mathbb{R}^{n}\right)$ and there holds

$\left(|x|^{m} V(x)\right)^{\prime} \leq 0$ in $\mathbb{R}^{n}, V=O\left(|x|^{-m}\right)$ and $\nabla V=O\left(|x|^{-m-1}\right)$ as $|x| \rightarrow \infty$.

C) $f(x, u) \in C^{1}\left(\mathbb{R}^{n} \times \mathbb{R}\right)$ and there exist $\Lambda>0$ and $m^{*}>\alpha^{\prime} \geq \alpha>$ $m(n-1) /(n-m)$ such that

$$
\Lambda^{-1} u^{\alpha-1} \leq f(x, u) \leq \Lambda u^{\alpha-1} \quad \text { for } \quad x \in \mathbb{R}^{n} \text { and } u>0,
$$

and

$$
\inf _{x \in \mathbb{R}^{n}, u>0} x \cdot \nabla_{x} F(x, u) \geq 0, \quad \nabla_{x} f(x, u)=O\left(|x|^{-1} f(x, u)\right),
$$

and

$$
\left(\alpha^{\prime}-1\right) f(x, u)-u f_{u}(x, u) \geq 0 \quad \Longrightarrow \quad \alpha^{\prime} F(x, u)-u f(x, u) \geq 0 .
$$

Then (1.1) does not admit any non-negative non-trivial solutions.

Remark. Note Theorem 5.3 applies to arbitrary non-negative solutions (not necessarily in $E$ ).

The proofs of Theorems 5.2 and 5.3 are based on the asymptotic estimates at infinity for positive solutions of (1.1) and the Pohozaev identity for (1.1).

Lemma 5.1. Assume the conditions of Theorem 5.2 hold. Then there exist positive constants $C=C\left(n, m, V_{0}\right)>0$ and $R=R(H, u)>0$ such that for $|x|>R$

$$
u(x) \leq C|x|^{-(n+m) / m}, \quad|\nabla u(x)| \leq C|x|^{-(n+m) / m}
$$

for all positive solutions $u \in E$ of (1.1).

Proof. For $u \in E$, without loss of generality, we assume

$$
H u^{m^{*}-m}(x)<K_{0}^{-1} / 2, \quad|x|>R_{0},
$$

since $u \rightarrow 0$ as $|x| \rightarrow \infty$. For $u \in E$ and $R>2 K$, multiply (1.1) by the test function $u \eta_{R}^{m}$ and integrate to obtain

$$
\int_{\mathbb{R}^{n}}|\nabla u|^{m} \eta_{R}^{m}+\int_{\mathbb{R}^{n}} V|u|^{m} \eta_{R}^{m} \leq M \int_{\mathbb{R}^{n}}|u|^{m}\left|\nabla \eta_{R}\right|^{m} \leq M R^{-m},
$$

where $M=M\left(K_{0}, m\right)>0$ is constant and $\eta_{R}=\eta(R /|x|)$, and we have used (5.2) and the Young inequality. Hence

$$
\int_{\mathbb{R}^{n}}|u|^{m} \eta_{R} \leq K_{0} \int_{\mathbb{R}^{n}} V|u|^{m} \eta_{R} \leq M K_{0} R^{-m}, \quad R>2 R_{0} .
$$

On the other hand, by (5.2) again, we have

$$
\Delta_{m} u \geq 0, \quad u>0 \quad \text { for }|x|>R_{0} .
$$

Hence the classical Harnack inequality (see $[11,15])$ together with (5.3) implies

$$
\sup _{B} u(x) \leq\left(\frac{C}{|B|} \int_{B}|u|^{m}\right)^{1 / m} \leq\left(C M K_{0}\right)^{1 / m}|z|^{-(n+m) / m},
$$


where $C=C(m, n)>0$ is constant and $B=B_{|z|}(z)$ with $|z|>3 R_{0}$. This yields $(5.1)_{1}$ with $R=2 R_{0}$. Using standard point-wise gradient estimates (see $[5,14])$, one derives $(5.1)_{2}$ from $(5.1)_{1}$.

To derive the asymptotic estimates under conditions of Theorem 5.3, we need the following Gidas-Spruck type inequality.

Lemma 5.2. Let $u$ be a positive $C^{1}$-solution of

$$
-\Delta_{m} u+V(x) u^{m-1}-f(x, u)-H(x) u^{m^{*}-1}=0 \quad \text { in } \mathbb{R}^{n} .
$$

Let $\phi \in C_{0}^{\infty}\left(\mathbb{R}^{n}\right)$ be a non-negative test function. Then for any $d \in \mathbb{R}$ there holds

$$
\begin{aligned}
& \int_{\mathbb{R}^{n}} \phi u^{1-m_{*}-d}|\nabla u|^{m}\left[A f(x, u)+A_{1} u f_{u}(x, u)-\left(A+(m-1) A_{1}\right) V u^{m-1}\right] \\
& +\left(A+\left(m^{*}-1\right) A_{1}\right) \int_{\mathbb{R}^{n}} \phi u^{1-m_{*}-d}|\nabla u|^{m} H u^{m^{*}-1}+B \int_{\mathbb{R}^{n}} \phi u^{-m_{*}-d}|\nabla u|^{2 m} \\
& \quad+A_{1} \int_{\mathbb{R}^{n}} \phi u^{1-m_{*}-d}|\nabla u|^{m-2} \nabla u\left[u \nabla_{x} f(x, u)-u^{m} \nabla V+u^{m^{*}} \nabla H\right] \\
& \leq \int_{\mathbb{R}^{n}} u^{1-m_{*}-d}\left[D\left(u f(x, u)-V u^{m}+H u^{m^{*}}\right)+D_{1}|\nabla u|^{m}\right]|\nabla u|^{m-2} \nabla u \cdot \nabla \phi \\
& \quad+\int_{\mathbb{R}^{n}} u^{2-m_{*}-d}\left(\nabla u \nabla^{2} \phi \nabla u\right)|\nabla u|^{2 m-4},
\end{aligned}
$$

where $m_{*}=m(n-1) /(n-m), D$ and $D_{1}$ are constants, $\nabla^{2} \phi$ is the Hessian of $\phi$ and

$$
A=\frac{n-1}{n}\left(1-\frac{d}{m_{*}}\right)\left(m^{*}-1\right), \quad A_{1}=-\frac{n-1}{n}, \quad B=\frac{m-1}{m} d(1-d) .
$$

This is precisely Proposition 6.1 of [12], by replacing $f(u)$ there by

$$
g(x, u)=f(x, u)+H u^{m^{*}-1}-V u^{m-1} .
$$

Utilizing Lemma 5.2, we have the following asymptotic estimates.

Lemma 5.3. Assume all conditions of Theorem 5.3 hold. Then there exist constants $C>0$ and $R>0$ such that

$$
u(x)=O\left(|x|^{-m /(\alpha-m)}\right), \quad \nabla u(x)=O\left(|x|^{-\alpha /(\alpha-m)}\right) ; \quad|x|>R,
$$

for all positive solutions of (1.1).

Proof. The proof is essentially the same as that of Theorem IV of [12]. We only sketch the proofs and the interested reader may find the details in [12].

Step 1. Use Lemma 5.2 to derive the key estimate

$$
\begin{aligned}
\int_{\mathbb{R}^{n}} \eta_{R}^{k} u^{2-m_{*}-d} f^{2}(x, u) \leq & C R^{-2 m} \int_{\mathbb{R}^{n}} \eta_{R}^{k-2 m} u^{2 m-m_{*}-d} \\
& +C R^{n-m\left(2 \alpha-m_{*}-d\right) /(\alpha-m)}
\end{aligned}
$$


where $\eta_{R}=\eta(|x-z| / R)$ with $|z|=3 R>0$ large. The arguments (Lemma 3.2) of [12] carry over, since all additional terms appeared in (5.4) can be properly dominated for $|x|>0$ large by using the assumptions and the boundary condition

$$
\lim _{|x| \rightarrow \infty} u(x)=0 .
$$

Also note

$$
A f(x, u)+A_{1} u f_{u}(x, u)-\left(A+(m-1) A_{1}\right) V u^{m-1} \geq C f(x, u) .
$$

We leave the details to the reader.

Step 2. Use the estimate (5.6) and the fact

$$
f(x, u)-V u^{m-1}+H u^{m^{*}-1}>0, \quad|x|>>1,
$$

to derive

$$
\int_{\mathbb{R}^{n}} \eta_{R}^{k} u^{2-m_{*}-d} f^{2}(x, u) \leq C R^{n-m\left(2 \alpha-m_{*}-d\right) /(\alpha-m)}
$$

for $R>0$ large, see (3.17) of [12].

Step 3. Derive the Harnack inequality

$$
\sup _{x \in B} u(x) \leq C \inf _{x \in B} u(x),
$$

where $B=B_{R}(z)$ with $|z|=3 R>0$ large. Again the arguments of Theorem 4.2 of [12] carry over by using the assumptions and the results in Step 2.

Step 4. (5.5) $)_{1}$ follows from Steps 2 and 3 immediately, while $(5.5)_{2}$ follows from the standard gradient point-wise estimates, with the aid of $(5.5)_{1}$.

Finally, we also use the following well-known Pohozaev type identity.

Lemma 5.4. Let $B=B_{R}(0)$ be the ball centered at the origin with radius $R>0$ and let $u$ be a solution of

$$
-\Delta_{m} u+V(x)|u|^{m-2} u-f(x, u)-H(x)|u|^{m^{*}-2} u=0 \quad \text { in } B .
$$

Then there holds

$$
\begin{aligned}
& \frac{1}{m} \int_{B}|x|^{1-m}|u|^{m}\left(|x|^{m} V\right)^{\prime}-\frac{1}{m^{*}} \int_{B}(x \cdot \nabla H)|u|^{m^{*}} \\
& -\int_{B} x \cdot \nabla_{x} F(x, u)+n \int_{B}\left[u f(x, u) / m^{*}-F(x, u)\right] \\
= & \int_{\partial B}|x|\left(|\nabla u|^{m} / m+V|u|^{m} / m-H|u|^{m^{*}} / m^{*}-F(x, u)\right) \\
& -\int_{\partial B}|x|^{-1}|\nabla u|^{m-2}\left[(x \cdot \nabla u)^{2}+(n-m)(x \cdot \nabla u) u / m\right] .
\end{aligned}
$$


Lemma 5.4 follows directly from Proposition 1 of [9] by taking $\Omega=B$ and $\mathcal{F}(x, u, p)=\frac{1}{m}|p|^{m}+\frac{1}{m} V|u|^{m}-F(x, u)-\frac{1}{m^{*}} H|u|^{m^{*}}, \quad h(x)=x, \quad a=\frac{n-m}{m}$.

Now we are ready to prove Theorems 5.2 and 5.3.

Proof of Theorem 5.2. We argue by contradiction. Let $u \geq 0$ be a non-trivial $E$-solution of (1.1), then $u$ is strictly positive by the classical strong maximum principle, see [16]. By (5.1) and the assumptions, one readily infers that

$$
\lim _{R \rightarrow \infty} \int_{\partial B}|x|\left(|\nabla u|^{m} / m+V|u|^{m} / m-H|u|^{m^{*}} / m^{*}\right)=\lim _{R \rightarrow \infty} O\left(R^{-m}\right)=0,
$$

and

$\lim _{R \rightarrow \infty} \int_{\partial B}|x|^{-1}|\nabla u|^{m-2}\left[(x \cdot \nabla u)^{2}+(n-m)(x \cdot \nabla u) u / m\right]=\lim _{R \rightarrow \infty} O\left(R^{1-m}\right)=0$.

With the help of Lemma 5.4, one then deduces

$$
\frac{1}{m} \int_{\mathbb{R}^{n}}|x|^{1-m}|u|^{m}\left(|x|^{m} V\right)^{\prime}-\frac{1}{m^{*}} \int_{\mathbb{R}^{n}}(x \cdot \nabla H)|u|^{m^{*}}=0 .
$$

In turn

$$
\int_{\mathbb{R}^{n}}|x|^{1-m}|u|^{m}\left(|x|^{m} V\right)^{\prime}=0 \quad \Longrightarrow \quad u(x) \equiv 0, \quad x \in \mathbb{R}^{n} .
$$

This is a contradiction and completes the proof.

Proof of Theorem 5.3. The proof is similar to that of Theorem 5.2. By (5.5) and the assumptions, one readily infers that

$$
\begin{aligned}
& \lim _{R \rightarrow \infty} \int_{\partial B}|x|\left(|\nabla u|^{m} / m+V|u|^{m} / m-H|u|^{m^{*}} / m^{*}-F(x, u)\right) \\
= & \lim _{R \rightarrow \infty} O\left(R^{n-m \alpha /(\alpha-m)}\right)=0,
\end{aligned}
$$

since $n-m \alpha /(\alpha-m)<0$ and

$$
\begin{aligned}
& \lim _{R \rightarrow \infty} \int_{\partial B}|x|^{-1}|\nabla u|^{m-2}\left[(x \cdot \nabla u)^{2}+(n-m)(x \cdot \nabla u) u / m\right] \\
= & \lim _{R \rightarrow \infty} O\left(R^{n-m \alpha /(\alpha-m)}\right)=0 .
\end{aligned}
$$

Hence by Lemma 5.4, we infer that there exists $C>0$ such that

$$
\begin{aligned}
C \int_{\mathbb{R}^{n}} u^{\alpha} & \leq C \int_{\mathbb{R}^{n}} F(x, u) \leq n \int_{\mathbb{R}^{n}}\left[F(x, u)-u f(x, u) / m^{*}\right] \\
& =\int_{\mathbb{R}^{n}}\left[|x|^{1-m}|u|^{m}\left(|x|^{m} V\right)^{\prime} / m-(x \cdot \nabla H)|u|^{m^{*}} / m^{*}-x \cdot \nabla_{x} F(x, u)\right] \\
& \leq 0,
\end{aligned}
$$

since the integrand is non-positive. Hence

$$
u(x) \equiv 0, \quad x \in \mathbb{R}^{n},
$$


again a contradiction.

To conclude this section, we would like to point out that one readily produces concrete examples which verify conditions given in Corollaries 4.1-4.3, and in Theorems 5.1-5.3, giving rise to existence and non-existence accordingly.

Acknowledgement. The author wishes to thank the referee(s) for their careful reading of the manuscript and their useful comments.

\section{References}

[1] A. Ambrosetti and P. Rabinowitz, Dual variational methods in critical point theory and applications, J. Functional Analysis 14 (1973), 349-381.

[2] T. Aubin, Nonlinear Analysis on Manifolds. Monge-Ampere Equations, Springer-Verlag, New York, 1982

[3] M.-F. Bidaut-Veron and S. Pohozaev, Nonexistence results and estimates for some nonlinear elliptic problems, J. Anal. Math. 84 (2001), 1-49.

[4] H. Brezis and L. Nirenberg, Positive solutions of nonlinear elliptic equations involving critical Sobolev exponents, Comm. Pure Appl. Math. 36 (1983), no. 4, 437-477.

[5] E. DiBenedetto, $C^{1+\alpha}$ local regularity of weak solutions of degenerate elliptic equations, Nonlinear Anal. 7 (1983), no. 8, 827-850.

[6] Y. Ding and F. Lin, Solutions of perturbed Schrodinger equations with critical nonlinearity, Calc. Var. Partial Differential Equations 30 (2007), no. 2, 231-249.

[7] P. L. Lions, The concentration-compactness principle in the calculus of variations. The limit case. I, Rev. Mat. Iberoamericana 1 (1985), no. 1, 145-201.

[8] _ The concentration-compactness principle in the calculus of variations. The limit case. II, Rev. Mat. Iberoamericana 1 (1985), no. 2, 45-121.

[9] P. Pucci and J. Serrin, A general variational identity, Indiana Univ. Math. J. 35 (1986), no. 3, 681-703.

[10] P. Rabinowitz, Minimax Methods in Critical Point Theory with Applications to Differential Equations, CBMS Regional Conference Series in Mathematics, 65. Published for the Conference Board of the Mathematical Sciences, Washington, DC; by the American Mathematical Society, Providence, RI, 1986.

[11] J. Serrin, Local behavior of solutions of quasi-linear equations, Acta Math. 111 (1964), $247-302$.

[12] J. Serrin and H. Zou, Cauchy-Liouville and universal boundedness theorems for quasilinear elliptic equations and inequalities, Acta Math. 189 (2002), no. 1, 79-142.

[13] G. Talenti, Best constant in Sobolev inequality, Ann. Mat. Pura Appl. (4) 110 (1976), 353-372.

[14] P. Tolksdorf, Regularity for a more general class of quasilinear elliptic equations, J. Differential Equations 51 (1984), no. 1, 126-150.

[15] N. Trudinger, On Harnack type inequalities and their application to quasilinear elliptic equations, Comm. Pure Appl. Math. 20 (1967), 721-747.

[16] J.-L. Vazquez, A strong maximum principle for some quasilinear elliptic equations, Appl. Math. Optim. 12 (1984), no. 3, 191-202.

[17] M. Willem, Minimax Theorems, Progress in Nonlinear Differential Equations and Their Applications, Vol. 24, Birkhäuser Boston, Inc., Boston, MA, 1996.

Department of Mathematics

University of Alabama at Birmingham

BiRMINGHAM, AL 35294, USA

E-mail address: zou@math.uab.edu 